\title{
Measuring the impact of observations on the predictability of the Kuroshio Extension in a shallow-water model
}

Article

Published Version

Kramer, W., Dijkstra, H. A., Pierini, S. and van Leeuwen, P. J. (2012) Measuring the impact of observations on the predictability of the Kuroshio Extension in a shallow-water model. Journal of Physical Oceanography, 42 (1). pp. 3-17. ISSN 0022-3670 doi: https://doi.org/10.1175/JPO-D-11-014.1 Available at https://centaur.reading.ac.uk/24130/

It is advisable to refer to the publisher's version if you intend to cite from the work. See Guidance on citing.

To link to this article DOI: http://dx.doi.org/10.1175/JPO-D-11-014.1

Publisher: American Meteorological Society

All outputs in CentAUR are protected by Intellectual Property Rights law, including copyright law. Copyright and IPR is retained by the creators or other copyright holders. Terms and conditions for use of this material are defined in the End User Agreement.

www.reading.ac.uk/centaur 
Central Archive at the University of Reading

Reading's research outputs online 


\title{
Measuring the Impact of Observations on the Predictability of the Kuroshio Extension in a Shallow-Water Model
}

\author{
Werner Kramer And Henk A. DiJKstra \\ Institute for Marine and Atmospheric Research Utrecht, Department of Physics and Astronomy, Utrecht University, \\ Utrecht, Netherlands \\ STEFANO PIERINI \\ Dipartimento di Scienze per l'Ambiente, Università di Napoli Parthenope, Naples, Italy \\ PETER JAN VAN LEEUWEN \\ Department of Meteorology, University of Reading, Reading, United Kingdom
}

(Manuscript received 10 January 2011, in final form 24 August 2011)

\begin{abstract}
In this paper, sequential importance sampling is used to assess the impact of observations on an ensemble prediction for the decadal path transitions of the Kuroshio Extension. This particle-filtering approach gives access to the probability density of the state vector, which allows the predictive power-an entropy-based measure - of the ensemble prediction to be determined. The proposed setup makes use of an ensemble that, at each time, samples the climatological probability distribution. Then, in a postprocessing step, the impact of different sets of observations is measured by the increase in predictive power of the ensemble over the climatological signal during one year. The method is applied in an identical-twin experiment for the Kuroshio Extension using a reduced-gravity shallow-water model. This study investigates the impact of assimilating velocity observations from different locations during the elongated and the contracted meandering states of the Kuroshio Extension. Optimal observation locations correspond to regions with strong potential vorticity gradients. For the elongated state the optimal location is in the first meander of the Kuroshio Extension. During the contracted state it is located south of Japan, where the Kuroshio separates from the coast.
\end{abstract}

\section{Introduction}

Our view of the oceans and atmosphere comes from remote sensing measurements and from still relatively sparse observations. Data assimilation combines the information gained from observations with computer simulations to obtain a three-dimensional representation of the current state of the ocean and atmosphere. An accurate estimate of the current state is particularly important for forecasting the future evolution. Because of the chaotic nature of these dynamical systems, uncertainties present in the initial state grow rapidly, reducing the utility of the

Corresponding author address: Werner Kramer, Institute for Marine and Atmospheric Research Utrecht, Dept. of Physics and Astronomy, Utrecht University, Princetonplein 5, 3584 CC Utrecht, Netherlands.

E-mail: w.kramer@uu.nl forecast. In this paper, we describe an ensemble-based method for measuring the effect of observations. Using model-generated velocity and sea surface height (SSH) observations, we apply the method to determine the best location for monitoring the decadal variability of the Kuroshio Extension (KE).

The Kuroshio Extension is the eastward-flowing free jet formed when the Kuroshio detaches from the Japanese coastline. The KE region has the largest SSH variability on subannual and decadal time scales in the extratropical North Pacific Ocean (Qiu 2002). The decadal variability is related to transitions of the KE from a highly energetic elongated path to a weaker contracted and more convoluted path (Qiu and Chen 2005). Prediction of the path of the Kuroshio is very important for local fisheries and hence local economies (Kagimoto et al. 2008). The position of the Kuroshio strongly determines the regions where phytoplankton and hence fish is located. The KE variability has 
been the subject of a number of experimental studies, like the observational programme of the Intergovernmental Oceanographic Commission (IOC) of the United Nations Educational, Scientific and Cultural Organization (UNESCO) sub-commission for the western Pacific (WESTPAC) at $152^{\circ} \mathrm{E}$ (Schmitz et al. 1982), the Kuroshio Extension Region Experiment (KERE) at $143^{\circ} \mathrm{E}$ (Hallock and Teague 1995), and the Kuroshio Extension System Study (KESS) at $146^{\circ} \mathrm{E}$ (Donohue et al. 2008).

Pierini (2006) obtained a reasonably successful comparison of satellite SSH observations and the results from a reduced-gravity shallow-water model of the western Pacific Ocean. The model is forced by a steady wind stress forcing and has only one active layer. In this model, not only the decadal transitions between contracted and elongated Kuroshio paths are found (Pierini et al. 2009), but the migration of the northward extension of the Kuroshio corresponds well with that determined from observations (Qiu and Chen 2005). Important to note is that the model also captures the high-frequency variability during the northward migration of the KE. The observed temporal variability is, however, higher than the modeled one and on smaller time scales. This is not surprising because the model does not capture baroclinic instability.

The physics behind the bimodality of the KE are not well understood and are still open for discussion. Pierini et al. (2009) explain the two states of the KE, its decadal period, and the changing high-frequency variability in terms of nonlinear dynamical systems theory. The success of the reduced-gravity shallow-water model suggests that the internal ocean mechanics are responsible. Another explanation is sought in the variable atmospheric forcing (Miller et al. 1998; Deser et al. 1999; Qiu 2003; Qiu and Chen 2005). Here, westward-propagating SSH anomalies, generated in the eastern North Pacific by wind stress anomalies at different phases of the Pacific decadal oscillation, cause the positional shifts of the KE jet.

Objective measures for the impact of observations on analysis uncertainties are important for both the design of persistent observation networks (e.g., mooring arrays) and the deployment of supplementary or targeted observations (e.g., airplane reconnaissance and dropsondes). Targeted observations are used to decrease the forecast uncertainty of high-risk events, like hurricanes. For an overview on targeted observations procedures and issues, see Langland (2005). An objective procedure, based on, for example, singular vectors, is used to determine regions with fast-growing initial errors. A targeted observation is considered, when the forecast error can be decreased by assimilating additional observation data. Baker and Daley (2000) argued that none of the traditional techniques consider the characteristics of the dataassimilation systems used. As such, interaction with the background field of the analysis and interactions with other observations are ignored. For this reason, objective procedures for adaptive observations are developed that incorporate the data-assimilation techniques (e.g., the ensemble transform Kalman filter; Bishop et al. 2001).

Objective procedures for targeted observations are not necessarily good for optimizing array designs. These methods aim to improve the forecast at a given time, whereas mooring arrays are operated over longer times. Sakov and Oke (2007) used an ensemble of system states from a long model run or from observation-based gridded fields to represent the time-averaged statistics of the system. The system states ensemble is used to obtain the background error covariances, which describe the uncertainty in the model state when no observational data is available. The optimal set of observations is then obtained by minimizing the analysis error covariance using Kalman filter theory. Problems arise when the timeaveraged statistics are strongly non-Gaussian, which makes an analysis based on covariances meaningless. As such, the bimodality of the Kuroshio Extension will present an obstacle for this method.

In this paper, we want to answer the question what is the best measurement location to monitor the Kuroshio Extension system for optimal prediction of its decadal transitions. The general idea is to perform an ensemble model forecast, which in our case is obtained with the reduced-gravity shallow-water model of Pierini (1996, 2006). The ensemble samples the time-averaged probability distribution of the system state. Observations are then used to update the ensemble using a particle-filtering technique. For this approach, neither the assumption of Gaussian error statistics nor the linearization of the model is required. The general methodology is described in section 2. The setup allows us to find which measurements are most successful in increasing the predictive skill or power of the ensemble forecast. In section 3 , results of an identical-twin experiment for the KE region are presented. We use this experiment to investigate the assets and drawbacks of the methodology. The test is done by assimilating velocity observations at four preselected locations. Thereafter, we use the method to find the optimal location for following the decadal transition in the KE. Final comments and conclusions are made in section 5.

\section{Methodology}

\section{a. Reduced-gravity shallow-water model}

In this paper, we utilize the reduced-gravity shallowwater model and setup used by Pierini (2006) to investigate the decadal oscillations of the Kuroshio Extension. The model describes the flow on a Cartesian grid in the upper layer with density $\rho$ of the ocean and assumes the second 


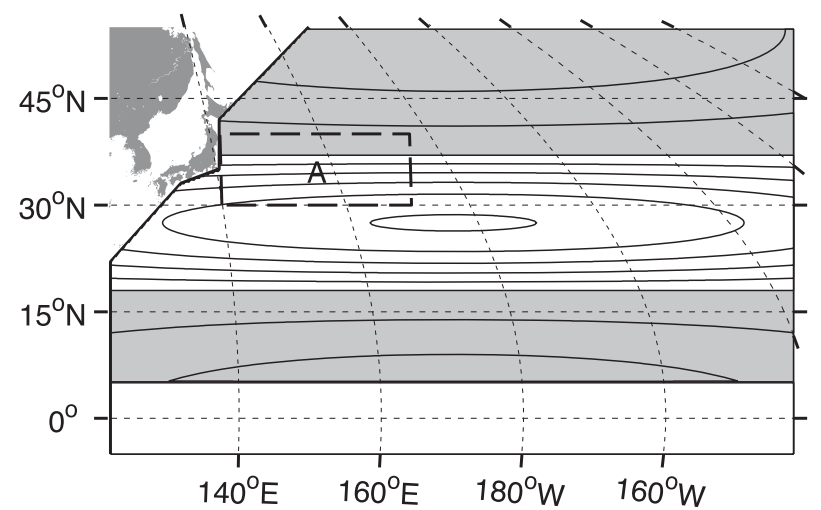

FIG. 1. The computational domain zonally spans $10700 \mathrm{~km}$ from $122^{\circ} \mathrm{E}$ to approximately $120^{\circ} \mathrm{W}$ at $35^{\circ} \mathrm{N}$ and ranges from $5^{\circ} \mathrm{S}$ to $55^{\circ} \mathrm{N}$ (Pierini 2006). At the western side, a schematic implementation of the Japanese coastline is used. The climatological wind stress curl is given by the contour map, with negative values in the light gray area (contour spacing is $1.5 \times 10^{-8} \mathrm{~N} \mathrm{~m}^{-3}$ ).

layer to be infinitely deep and quiescent. The shallowwater equations describing the flow in the upper layer are

$$
\begin{aligned}
\frac{\partial \mathbf{u}}{\partial t}+\mathbf{u} \cdot \nabla \mathbf{u}+f \mathbf{k} \times \mathbf{u}= & -g^{\prime} \nabla \eta+A_{H} \nabla^{2} \mathbf{u} \\
& +\frac{\boldsymbol{\tau}}{\rho h}-\gamma \mathbf{u}|\mathbf{u}| \text { and } \\
\frac{\partial h}{\partial t}+\nabla \cdot(h \mathbf{u})= & 0,
\end{aligned}
$$

where $\mathbf{u}=(u, v)$ is the horizontal velocity vector, $h$ is the upper-layer thickness, $\eta$ is the interface displacement (positive downward), and $\mathbf{k}$ is the unit vector in vertical direction.

The equations include the Coriolis term (the Coriolis parameter $f=2 \Omega \sin \phi$ at latitude $\phi$, where $\Omega$ is the earth's angular velocity) and a dissipation term with horizontal eddy viscosity $A_{H}$. The flow is driven by a wind stress $\tau$ exerted on the free surface. A density difference between the upper and lower layers results in a reducedgravity constant $g^{\prime}$. The friction between the two fluid layers is modeled by a quadratic stress weighted by the coefficient $\gamma$. At each time, the flow is fully described by the state vector $\mathbf{X}=(\mathbf{u}, h)$.

The specific domain Pierini (2006) used to model the $\mathrm{KE}$ is presented in Fig. 1. A schematic implementation of the Japanese coastline proved to be essential for capturing the dynamics of the KE (Pierini 2008). The flow is driven by a constant-in-time wind stress (with an amplitude of $5 \times 10^{-2} \mathrm{~N} \mathrm{~m}^{-2}$ ), which is an analytical approximation of the climatological double-gyre wind field. In our study, the wind stress field is perturbed by a stochastic wind field (with an amplitude of $2.3 \times 10^{-2} \mathrm{~N} \mathrm{~m}^{-2}$ ),
TABLE 1. Settings for the reduced-gravity shallow-water model. The equations are solved numerically on a Cartesian grid with spacing $(\Delta x, \Delta y)$ and time step $\Delta t$. The upper layer has a mean depth $H$ and density $\rho$. The friction between the upper and lower layers is quadratic with a friction coefficient $\gamma$.

\begin{tabular}{ll}
\hline \hline$\Delta x$ & $20 \mathrm{~km}$ \\
$\Delta y$ & $20 \mathrm{~km}$ \\
$\Delta t$ & $20 \mathrm{~min}$ \\
$A_{H}$ & $220 \mathrm{~m}^{2} \mathrm{~s}^{-1}$ \\
$H$ & $500 \mathrm{~m}$ \\
$\rho$ & $1023.5 \mathrm{~kg} \mathrm{~m}^{-3}$ \\
$g^{\prime}$ & $4.41 \times 10^{-3} \mathrm{~m} \mathrm{~s}^{-2}$ \\
$\gamma$ & $5.0 \times 10^{-4} \mathrm{~m}^{-1}$ \\
\hline
\end{tabular}

which is Gaussian correlated in space with a correlation length of $2000 \mathrm{~km}$. The amplitude of the stochastic wind field is uncorrelated in time (white noise). The parameter values can be found in Table 1, and further details on the model formulation and implementation are in Pierini (2006).

\section{b. Particle filtering}

The shallow-water model can be used to give a forecast for the Kuroshio Extension, when an initial state $\mathbf{X}_{0}$ is provided. Pierini et al. (2009) showed for realistic parameter values that the system has a positive Lyapunov exponent. The chaotic nature of the system limits the prediction time, as initial perturbations grow over time. The uncertainty in the initial state is assigned to probability density function ( $\mathrm{pdf}) p\left(\mathbf{X}_{0}\right)$. Moreover, the model is not an exact representation of reality as imperfections are present in the model equations, parameters, and boundary conditions. We model these uncertainties with stochastic perturbations to the wind stress field. The shallow-water model described in the previous section can be considered as a discrete time estimation problem. The evolution of the state vector $\mathbf{X}_{k}$ is described by the following system model:

$$
\mathbf{X}_{k+1}=M_{k}\left(\mathbf{X}_{k}, \zeta_{k}\right),
$$

where $M_{k}$ is the system's propagator and $\zeta_{k}$ is a zeromean, white-noise sequence.

Observations $\mathbf{Y}_{k}$ become available at discrete times. The aim of a data-assimilation technique is to obtain a conditional probability density function of the current state at $t=t_{k}$ given all available information, $p\left(\mathbf{X}_{k} \mid \mathbf{Y}_{1: k}\right)$. Taking a Monte Carlo approach, $p\left(\mathbf{X}_{k}\right)$ is randomly sampled by a weighted ensemble of $N$ model realizations $\mathbf{X}_{k}^{i}$ (also called particles),

$$
p_{N}\left(\mathbf{X}_{k}\right)=\sum_{i=1}^{N} w_{k}^{i} \delta\left(\mathbf{X}_{k}-\mathbf{X}_{k}^{i}\right),
$$


with weights $w_{k}^{i}$. The initial state of each ensemble member is uniformly drawn $\left(w_{0}^{i}=1 / N\right)$ from the initial pdf $p\left(\mathbf{X}_{0}\right)$. In a particle-filtering method, $p_{N}\left(\mathbf{X}_{k} \mid \mathbf{Y}_{1: k}\right)$ is obtained recursively in a prediction step and an update step. Assume that $p_{N}\left(\mathbf{X}_{k-1} \mid \mathbf{Y}_{1: k-1}\right)$ is known: that is, the particle states $\mathbf{X}_{k-1}^{i}$ and the weights $w_{k-1}^{i}$ are known. Now, the next observation $\mathbf{Y}_{k}$ becomes available. In the prediction step, each particle $\mathbf{X}_{k-1}^{i}$ is integrated forward in time to obtain the state vector at the new time $\mathbf{X}_{k}^{i}$. The probability $p_{N}\left(\mathbf{X}_{k} \mid \mathbf{Y}_{1: k-1}\right)$ follows from

$$
p_{N}\left(\mathbf{X}_{k} \mid \mathbf{Y}_{1: k-1}\right)=\sum_{i=1}^{N} w_{k-1}^{i} \delta\left(\mathbf{X}_{k}-\mathbf{X}_{k}^{i}\right) .
$$

In the update step, Bayes' theorem is exploited to get

$$
p_{N}\left(\mathbf{X}_{k} \mid \mathbf{Y}_{1: k}\right)=\frac{p\left(\mathbf{Y}_{k} \mid \mathbf{X}_{k}\right) p_{N}\left(\mathbf{X}_{k} \mid \mathbf{Y}_{1: k-1}\right)}{p\left(\mathbf{Y}_{k}\right)} .
$$

Inserting the pdf (3) for $p\left(\mathbf{X}_{k} \mid \mathbf{Y}_{1: k}\right)$ in Eq. (5) yields, for the new weights,

$$
w_{k}^{i}=\frac{p\left(\mathbf{Y}_{k} \mid \mathbf{X}_{k}^{i}\right)}{p\left(\mathbf{Y}_{k}\right)} w_{k-1}^{i} .
$$

Here, the probability of the observation $p\left(\mathbf{Y}_{k}\right)$ can be considered as a normalization factor. This normalization can also be accomplished by demanding that $\sum_{i=1}^{N} w_{k}^{i}=1$. The probability of the observation given the model $p\left(\mathbf{Y}_{k} \mid \mathbf{X}_{k}^{i}\right)$ is directly linked to the (known) observational error. For example, for an univariate measurement $Y_{k}$ with a Gaussian distribution for the measurement error (with standard deviation $\sigma_{\mathrm{obs}}$ ), this probability follows from $p\left(Y_{k} \mid \mathbf{X}_{k}\right) \sim \exp \left\{-\left[H\left(\mathbf{X}_{k}^{i}\right)-Y_{k}\right]^{2} / 2 \sigma_{\text {obs }}^{2}\right\}$. Here, $H\left(\mathbf{X}_{k}^{i}\right)$ is the model equivalent of the observation calculated using the observation operator $H$.

For an overview of particle filtering in geophysical systems the reader is referred to Van Leeuwen (2009). The particular particle filter method described here is known as sequential importance sampling (SIS) (see, e.g., Doucet et al. 2001). In contrast with other dataassimilation techniques, particle filtering does not require a linearization of the model around the current state, nor does it assume Gaussian statistics for the state variables. An advantage of SIS is that assimilating observations changes the weight but leaves the particle $\mathbf{X}_{k}^{i}$ itself unchanged. Hence, the ensemble can be run beforehand, and the impact of different sets of observations can be calculated afterward. A major problem of SIS is that after a number of observations the weight is concentrated on a small number of particles. The effective number of particles can be estimated by $N_{\text {eff }}=$ $1 / \sum_{i=1}^{N}\left(w_{k}^{i}\right)^{2}$. The traditional solution of this degeneracy of the ensemble is to resample $p\left(\mathbf{X}_{k} \mid \mathbf{Y}_{1: k}\right)$ with an altered set of particles. A common technique of resampling consists of making copies of particles with a high weight and discard particles with a low weight. Because of the stochastic forcing and chaotic dynamics, a particle and its copy will diverge over time and become uncorrelated. Resampling, however, breaks the desired property of evaluating observations as a postprocessing step after the time integration of the ensemble is completed.

\section{c. Entropy-based predictability measures}

The application of the particle filter method allows us to sample the non-Gaussian probability distribution of the state vector. To specify the amount of uncertainty in the probability density function, entropy-based measures are favorable. In this paper, we use two measures that are adopted to quantify the predictability of an ensemble forecast. The first is the predictive power (PP) introduced by Schneider and Griffies (1999), which is a measure of the uncertainty relative to the climatological variance. The second is the potential prediction utility (PPU), as introduced by Kleeman (2002), which additionally incorporates a signal-to-noise component in the measure (see also Xu 2006).

The predictive power is based on the entropy $S_{p(\mathbf{X})}$, a measure for the uncertainty associated with the pdf $p(\mathbf{X})$ of variable $\mathbf{X}$ (Shannon 1948). The differential entropy is defined as

$$
S_{\mathbf{X}} \equiv-\kappa \int p(\mathbf{X}) \ln p(\mathbf{X}) d \mathbf{X}
$$

Here, $\kappa$ is a constant that determines the unit of entropy. The particle filtering allows one to obtain an approximation to the probability density function $p\left(\mathbf{X}_{k} \mid \mathbf{Y}_{1: k}\right)$ of the state vector. With the pdf available, the entropy $S_{p(\mathbf{x})}$ of the ensemble forecast can be calculated. Schneider and Griffies (1999) defined the predictive power $\alpha_{\mathbf{X}}$ of an ensemble forecast as

$$
\alpha_{\mathbf{X}} \equiv 1-\exp \left(-S_{q(\mathbf{X})}+S_{p(\mathbf{X})}\right)
$$

The entropy $S_{q(\mathbf{X})}$ is calculated from the pdf of the climatology $q(\mathbf{X})$, and it can be considered as the uncertainty when only the climatological mean is known. Note that differential entropy (7) is not scaling invariant and cannot be compared directly to the discrete entropy. Two differential entropies can be compared as long as the reference scales are the same. The PP is limited to the range $0 \leq \alpha_{\mathbf{X}} \leq 1$. If the ensemble analysis is equal to the climatological mean $\left[S_{p(\mathbf{x})}=S_{q(\mathbf{x})}\right]$, its PP is zero. When the entropy of the ensemble forecast reduces 
(e.g., by assimilating observations), the predictive power will increase. Note that because of the exponential form PP is most sensitive in the lower part in the scale, if $S_{p(\mathbf{X})}$ is close to $S_{q(\mathbf{X})}$. Schneider and Griffies (1999) defined the predictive power to measure the decrease in uncertainty of the full state vector and set $\kappa=1 / m$ with $m$ the dimension of the state vector. This makes it possible to compare entropies of random state vectors of different dimensions.

The potential prediction utility is based on the relative entropy for $p(\mathbf{X})$,

$$
R_{\mathbf{X}} \equiv \int p(\mathbf{X}) \ln \frac{p(\mathbf{X})}{q(\mathbf{X})} d \mathbf{X}
$$

which is the information gain over the reference pdf $q(\mathbf{X})$. The relative entropy for a continuous pdf is scaling invariant. For the predictive utility, this reference distribution is the climatological or equilibrium probability distribution. Like the predictive power, the PPU is zero when the probability distribution of the analysis is equal to the climatological distribution. The PPU of the analysis increases when the spread in probability distribution decreases, but also when the analysis relates to a relatively rare event. There is no theoretical upper limit for the PPU. Kleeman and Majda (2005) point out that PPU calculated over the full state vector $R_{\mathbf{X}}$ decreases monotonically over time as uncertainty increases (see also Cover and Thomas 1991).

In this paper, we opt to calculate the predictive power $\alpha_{s}$ for a single scalar quantity $s$ (e.g., the kinetic energy $E$ integrated over the KE region), following Pierini (2006). The reasoning behind determining the predictive power for the kinetic energy is that one is not always interested in reducing the uncertainty in the full state vector space. Our design aim is to follow the path transitions of the Kuroshio Extension and hence we optimize for the kinetic energy in the KE region. The entropies $S_{q}(s)$ and $S_{p}(s)$ are calculated from the univariate pdf $q(s)$ and $p(s)$ and we set $\kappa=$ 1 in (7). If $p(s)$ and $q(s)$ are Gaussian probability distributions with variances $\sigma_{p}^{2}$ and $\sigma_{q}^{2}$, the predictive power is given by $\alpha_{s}=1-\sigma_{p}^{2} / \sigma_{q}^{2}$. This formulation (or its multivariate counterpart) is often used in predictability studies that are based on the analysis error covariance.

\section{d. Setup of the identical-twin experiment}

Instead of forecasting the actual Kuroshio Extension by assimilating real observations in the shallow-water model, an identical-twin experiment will be performed. Here, one model realization is considered to be the "true" evolution of the KE. Equivalent observations are produced by taking measurements from this synthetic truth and adding observational errors. This allows us to produce observations for different variables, like the SSH or the velocity field, with different error distributions. Then, an analysis is performed by assimilating the observations in an ensemble run of the shallow-water model using the particle-filtering technique.

The advantage of the identical-twin experiment is that we are sure that the evolution of the true KE is captured by the ensemble run with the shallow-water model. A failure to describe the truth is then not caused by the model, but is caused by either insufficient observations or the inadequacy of the data-assimilation method.

In this study, we use a particular setup for the ensemble. At each time, the ensemble samples the climatological probability density function $q(\mathbf{X})$. This is achieved by drawing the initial conditions $\mathbf{X}_{0}^{i}$ from the climatological distribution. In practice, we obtained the initial states by taking snapshots, 2 yr apart, of the state vector from a long model run. For each particle $(N=512)$ the shallow-water model driven by the stochastic wind stress is integrated over 40 yr.

Assimilating the synthetic observations using the particle filter (6) will change the weight of the ensembles members. Starting with a uniform distribution of the weights $\left(w_{0}^{i}=1 / N\right)$, the initial probability distribution is equal to the climatology probability distribution. This initial state has zero predictive power and zero potential prediction utility. Hence, any increase of the PP of the ensemble is due to the information the observations provide.

\section{The impact of observations on the predictability}

\section{a. The predictive power of an ensemble forecast}

As an illustrative example, we first use the predictive power and potential prediction utility to determine the predictability time of an ensemble prediction with the shallow-water model under stochastic wind forcing. Essentially, this is a predictability study of the second kind (Lorenz 1975), where the influence of uncertain boundary conditions - in our case in the wind stress - on the predictability is determined. For this purpose, we have run a 96-member ensemble that starts from identical initial conditions. The initial state $\mathbf{X}_{0}^{i}$ is obtained from a 50-yr spinup model run. With no uncertainty present in the initial conditions (i.e., the differential entropy is infinite) the ensemble starts with a predictive power equal to unity. This is similar to the setup used by Griffies and Bryan (1997) to study the predictability of the North Atlantic multidecadal climate variability. Their study then motivated Schneider and Griffies (1999) to introduce the predictive power as a measure for the uncertainty in ensemble model forecasts. 
a) kinetic energy of all ensemble members

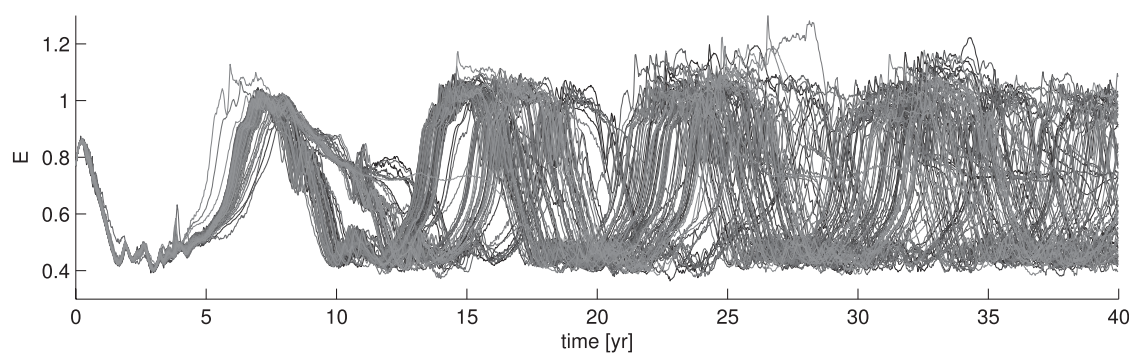

b) predictive power

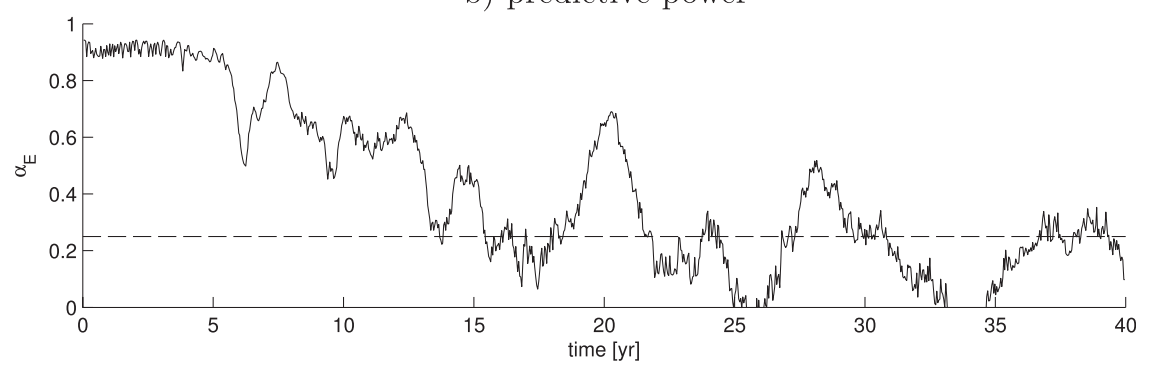

FIG. 2. Ensemble forecast for the KE with a stochastic forced shallow-water model starting from a single initial state. (a) Evolution of the kinetic energy $E$ of the KE for the 96 ensemble realizations and (b) PP $\alpha_{E}$ for the kinetic energy signal of the forecast.

In Fig. 2a, the time evolution of the kinetic energy of the Kuroshio Extension is plotted. This kinetic energy is defined by $E=(1 / 2) \int_{A}|\mathbf{u}|^{2} d S$, where $A$ is the region of the free jet as defined in Fig. 1. The energy signal clearly reveals the decadal transitions of the KE between a lowenergy state and a high-energy state. The two KE states are less clear from time series of potential vorticity or enstrophy integrated over the same region. With knowledge of the kinetic energy, one could already provide a good estimate for the pathlength and the mean latitude of the jet (Pierini et al. 2009). The first strong divergence of the ensemble occurs after $5 \mathrm{yr}$, when the transition from the low-energy state to the high-energy state starts. Then, after $8 \mathrm{yr}$, the signals branch. The main branch relates to a fast transition to the low-energy state, whereas the other branch reveals a slow decay of the kinetic energy for 2, 4, or $6 \mathrm{yr}$. As the signals become desynchronized, the predictability of the system is limited to a certain time period.

In Fig. $2 b$, the predictive power of the ensemble is given for a time span of $40 \mathrm{yr}$. Here, the PP (8) is calculated using $p(E)$ instead of the probability density function $p(\mathbf{X})$ of the full state vector. This indicates that we investigate the PP of the energy signal and not of the complete information the forecast provides. An estimate for the climatology pdf $q(E)$ is obtained by binning the time series of the $N=512$-particle ensemble (described in the previous section) over 23 bins. The approximation for $p(E)$ is obtained over the same bins. Note that the maximum $\mathrm{PP}$ is limited by the bin spacing $\Delta E$ as the minimum entropy $S_{P(E)}=\ln \Delta E$ (Cover and Thomas 1991): that is, when all the particles fall within one bin.

During the first $5 \mathrm{yr}$, the predictive power in Fig. $2 \mathrm{~b}$ remains high. Then, over a one-year period the predictive power drops to $\alpha_{E}=0.5$. Subsequently, the PP rises and ranges between $\alpha_{E}=0.6$ and 0.8 for years $6-13$. This rise of $\alpha_{E}$ is the result of the energy plateaus in the signal. This causes a smaller uncertainty in $E$, while the signals clearly become desynchronized. In this period, the weighted ensemble pdf for $E$ is strongly bimodal or trimodal. Schneider and Griffies (1999) defined the predictability life span as the lead time for which the $95 \%$ confidence interval of the predictive power does not include zero. This requires detailed information on the error in the prediction probability distribution $p(E)$, which is not available from one ensemble. In reality, an ensemble prediction loses its usefulness at an earlier time, because there are more strict requirements for the forecast uncertainty. For simplicity, we define the predictability life span of the ensemble as the time $\alpha_{E}$ drops below 0.25 . This definition results in a predictability life of $13 \mathrm{yr}$. During this time, it is possible to predict whether the KE is in the high-energy state or in one of the other states.

\section{b. Measuring the impact of observations}

Now, we return to our objective of measuring the impact of observations. For this purpose, we solely use the 512-particle ensemble, which samples the climatology 

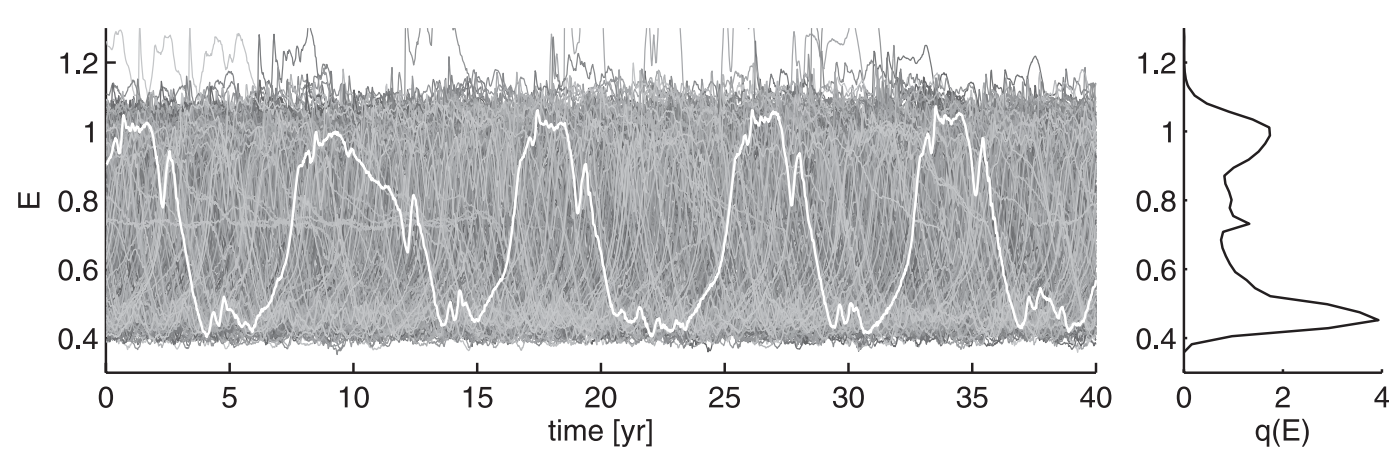

FIG. 3. Data from the ensemble run for the identical-twin experiment. The gray lines indicate the kinetic energy $E$ for the 511 particles, whereas the white curve corresponds to the synthetic truth. (right) The climatological pdf $q(E)$ is given.

distribution at any time. The energy evolution for all the particles is given in Fig. 3. The unweighted mean of this ensemble is always equal to the climatological mean field, and its predictive power is equal to zero. Clearly, the climatological pdf is bimodal. As the analysis starts from the climatological distribution, a data-assimilation method that can handle non-Gaussian statistics is required.

One particle is selected and is considered to be the true behavior of the Kuroshio Extension (e.g., the white curve in Fig. 3). From this synthetic truth, almost any observation can be produced, because we have access to the full state vector. For now, we opt to limit our studies to four different locations, or moorings, where observations of both velocity components are obtained. The velocity components are directly available from the shallow-water model. For this setup, the observation operator $H\left(\mathbf{X}_{k}^{i}\right)$ simply selects the velocity components from the state vector at the mooring location.

The selection of the four locations is based on the climatological mean and variance of the SSH (see Fig. 4). In practice, sea surface height fields are readily available from satellite observations. The moorings are located in the circulation cell to the south of Japan (A) and at the positions where the local maxima of SSH variance occur (B, C, and D). Here, mooring B is located at the absolute maxima, mooring $\mathrm{C}$ is in the $\mathrm{KE}$ near the first meander, and mooring $\mathrm{D}$ is at a more eastern location in the $\mathrm{KE}$ region. The synthetic velocity observations for the four moorings are given in Fig. 5. Observations for the zonal and meridional velocity components are produced at a monthly data rate (each 28 days) and have a Gaussian measurement error with a standard deviation of $\sigma_{\mathrm{obs}}=$ $0.1 \mathrm{~m} \mathrm{~s}^{-1}$. Observation errors for the zonal and meridional velocity are not correlated; as such they can be assimilated sequentially.

With the particle-filtering technique (SIS) described in section 2, the observations are used to improve our ensemble forecast. Cycling through the observations in a sequential order, the weights of each particle are adjusted according to Eq. (6). The resulting analysis is the weighted ensemble of all the particles. The probability density function $p(E)$ of the analysis is given in Fig. 6. It becomes immediately clear that observations from mooring $\mathrm{B}$ are most effective in reducing the uncertainty. With observations from mooring $\mathrm{C}$ we cannot determine whether the $\mathrm{KE}$ is in the high- or low-energy state for the first $3 \mathrm{yr}$. Assimilating data from mooring A rightly puts the system in the high-energy state, but with a large uncertainty in the initial years. If the first $\mathrm{KE}$

a) sea-surface height

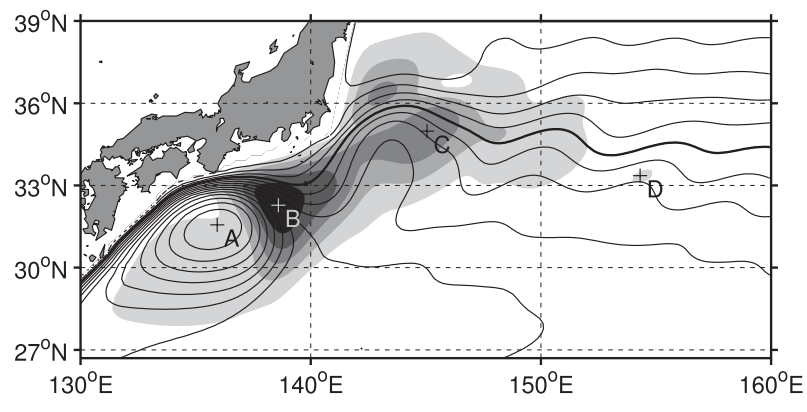

b) potential vorticity

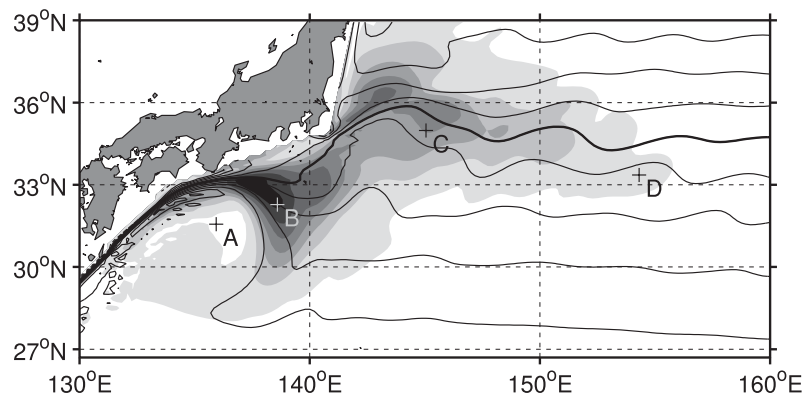

FIG. 4. (a) Climatological mean SSH field and (b) PV calculated from the ensemble. The gray scales represent the standard deviation with respect to the climatological mean. Contour spacing is $5 \mathrm{~cm}$ for the SSH and $10^{-8} \mathrm{~s}^{-1} \mathrm{~m}^{-1}$ for the PV. The thick contours give the 10-cm SSH level and the $1.6 \times 10^{-7} \mathrm{~s}^{-1} \mathrm{~m}^{-1} \mathrm{PV}$ level. Observations are produced for the positions labeled A, B, C, and D. 

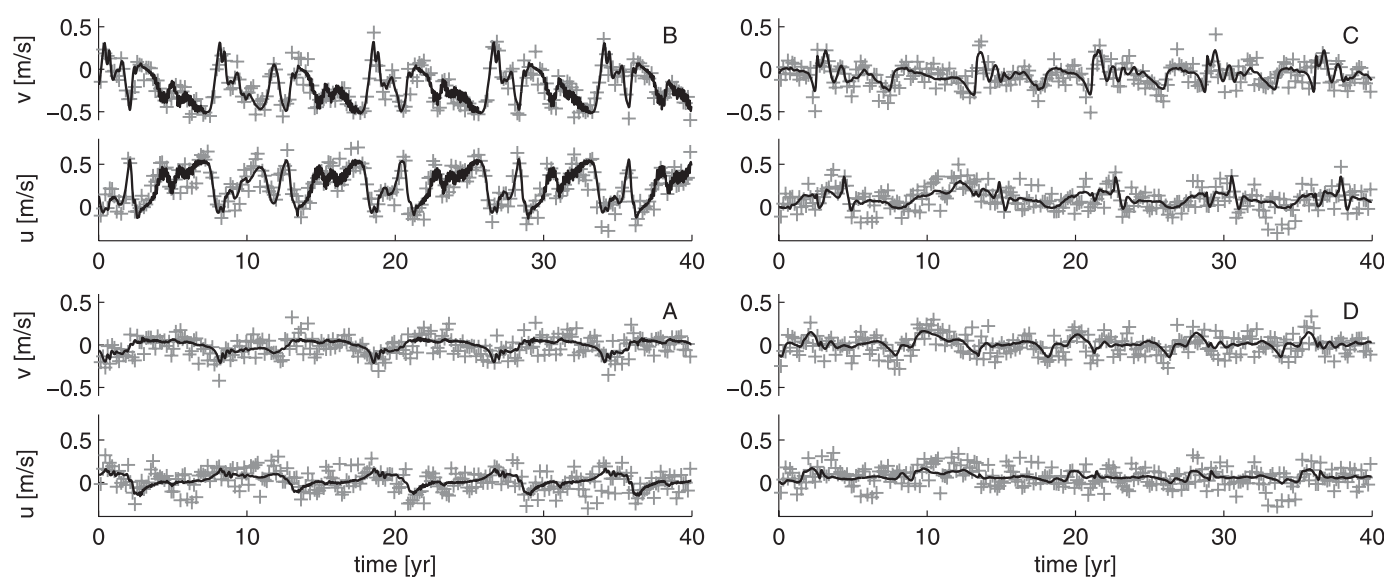

FIG. 5. Observations (plus signs) for the identical-twin experiment are produced by adding a Gaussian measurement error to the synthetic truth (drawn). Observations for $u$ and $v$ are produced for the four locations A, B, C, and D as shown in Fig. 4.

transition occurs, results improve for moorings A and C. The analysis using data from mooring $\mathrm{D}$ is the worst. During the first $10 \mathrm{yr}$, we are unable to determine the state of the KE from the analysis.

For times longer than $3 \mathrm{yr}$, the ensemble becomes degenerated for all cases. Then the analysis does not capture the true behavior nor does it give an accurate representation of the probability distribution. The observations at location B have a high signal-to-noise ratio. The first few observations will rapidly reduce the uncertainty from its initial value. As a consequence, a large number of particles is discarded after each observation and the ensemble rapidly degenerates. In Fig. 7, the effective number of particles $N_{\text {eff }}$ is given for the four moorings. For mooring B all the weight effectively collapses to a few particles after $2 \mathrm{yr}$. The analysis still follows the first transitions of the KE, because of the slow divergence of particle trajectories: the predictability life is $13 \mathrm{yr}$. One particle close to the truth will follow the true evolution for a few years. A degenerated ensemble does not accurately capture the real uncertainty, and it is not guaranteed that it can track the true evolution. For instance, the degenerated analysis for mooring B fails to describe the KE transitions between years 12 and 20.

At each time, a discrete probability distribution for the kinetic energy can be obtained by binning the weighted ensemble. The number of bins used to obtain the histogram of $p(E)$ and $q(E)$ is $\sqrt{N}$. The probability density $p_{j}(E)$ is the sum over the weights from particles that fall in bin $j$ divided by the bin size $\Delta E$. An estimate is obtained for $S_{P(E)}($ with $\kappa=1)$ by numerical integration over the discrete histogram: that is, $(1 / \kappa) \sum_{j} p_{j}(E) \ln p_{j}(E) \Delta E$. The
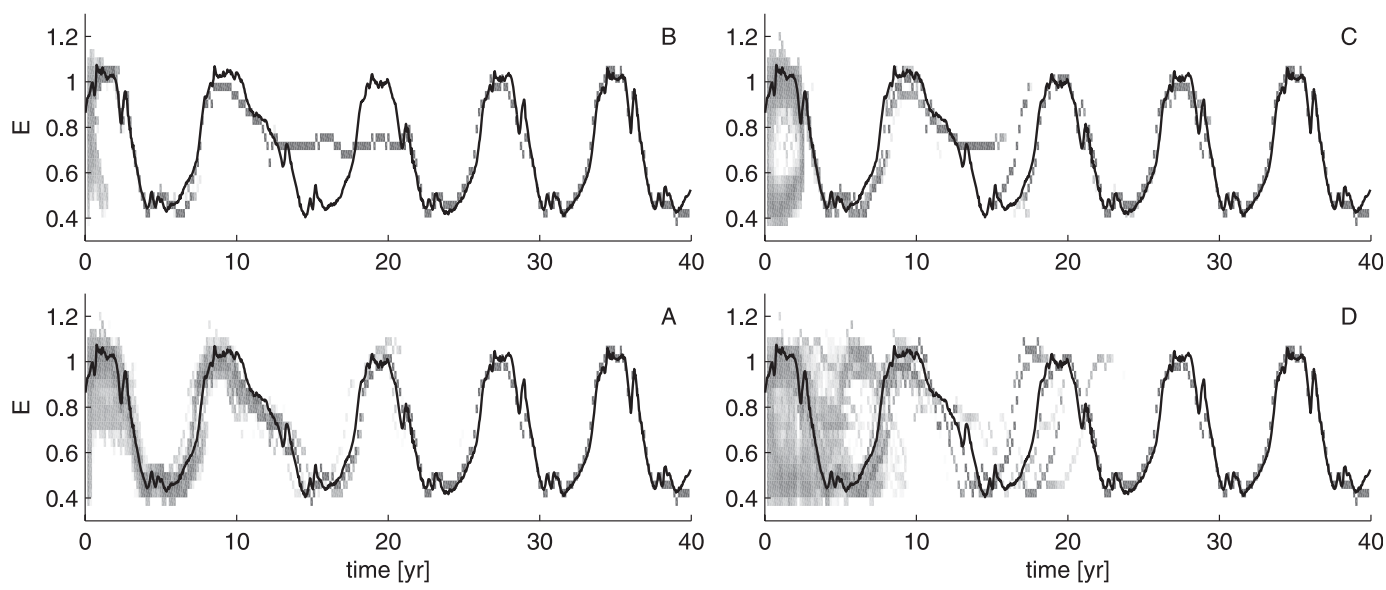

FIG. 6. Analysis of the kinetic energy $E$ of the KE resulting from assimilating velocity observations from location $\mathrm{A}, \mathrm{B}, \mathrm{C}$, or D. The grayscale plot gives $p(E)$ of the ensemble analysis, with the darkest gray related to highest probability density. The black line corresponds with the synthetic truth. 

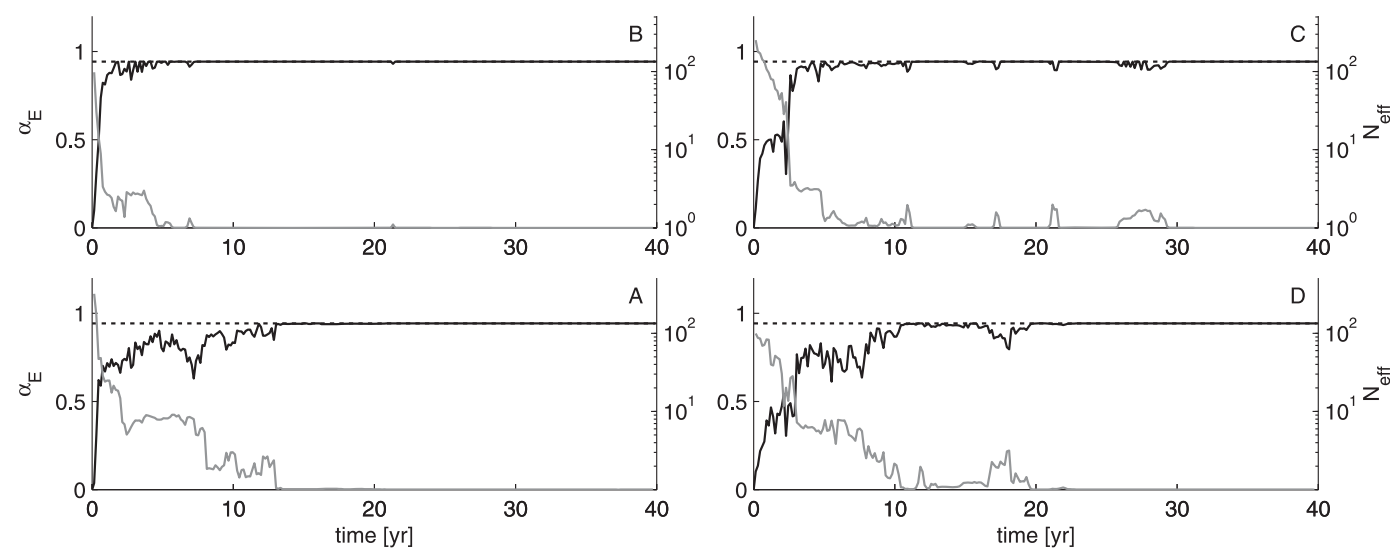

FIG. 7. PP $\alpha_{E}$ (black line) for the analysis (given in Fig. 6) resulting from assimilating velocity observations from location A, B, C, or D. The limit on the PP due to the used bin size is depicted by the dotted line. The effective number of particles $N_{\text {eff }}$ is denoted by the gray curve.

unweighted ensemble gives an estimate for the climatology pdf of $S_{q(E)}=-0.45$. The predictive power $\alpha_{E}$ is given in Fig. 7. The graph nicely shows that assimilating observations from mooring $\mathrm{B}$ raises the predictive power of the ensemble rapidly from zero to its limiting value after 3 yr. Note that reaching the limiting value for PP means that all the particle weights fall within one bin. Observations from the other mooring raise the predictive power at a slower rate. For moorings $\mathrm{C}$ and $\mathrm{D}$, the sudden increase in predictive power is related to the transition from the high-energy state to the low-energy state. This gives a clear signal in the velocity at these locations.

Although a single analysis gives already much insight into the impact of different observations, a number of problems arise. The selected synthetic truth might show some sporadic, unrepresentative behavior. Moreover, this synthetic truth starts from a state that might be more or less sensitive to perturbations. This can produce a different initial increase of the predictive power. Picking another particle to act as the synthetic truth, creating new observations, and performing a new analysis will give different results. We exploit this by selecting each of the 512 model realizations to be the synthetic truth at a time. This leads to 512 different evolutions of the entropy $S_{P(E)}$. The average entropy $\bar{S}_{p(E)}$ is then used to define the predictive power $\bar{\alpha}_{E}=1-\exp \left(-S_{q(E)}+\bar{S}_{p(E)}\right)$. In Fig. 8, the predictive power is given up to $20 \mathrm{yr}$. By assimilating data from mooring $\mathrm{B}$, the predictive power rapidly increases to a value of 0.7 in one year. For mooring $\mathrm{C}$ the same $\bar{\alpha}_{E}$ is reached after $2 \mathrm{yr}$, whereas $5 \mathrm{yr}$ are required when assimilating data from mooring $\mathrm{A}$ or $\mathrm{D}$.

In the special context of sequential importance sampling and the specific discrete approximations (3) for $p(\mathbf{X})$ and $q(\mathbf{X})$, there is an accurate way of calculating the PPU for the full state vector. For an ensemble where the initial states are drawn from $q(\mathbf{X})$, the discrete approximation is
$q_{N}\left(\mathbf{X}_{0}\right) \sum_{i=1}^{N} w_{0}^{i} \delta\left(\mathbf{X}_{0}-\mathbf{X}_{0}^{i}\right)$, with $w_{0}^{i}=1 / N$. If this ensemble is integrated forward in time, it still represents the climatological pdf; that is, $q_{N}\left(\mathbf{X}_{k}\right)=\sum_{i=1}^{N} w_{0}^{i} \delta\left(\mathbf{X}_{k}-\mathbf{X}_{k}^{i}\right)$ is also an approximation of $q(\mathbf{X})$. Assimilating $k$ observations changes the weights and an approximation for $p\left(\mathbf{X}_{k} \mid \mathbf{Y}_{1: k}\right)$ is given by $p_{N}\left(\mathbf{X}_{k} \mid \mathbf{Y}_{1: k}\right)=\sum_{i=1}^{N} w_{k}^{i} \delta\left(\mathbf{X}_{k}-\right.$ $\mathbf{X}_{k}^{i}$ ). Inserting these approximations in (9) yields

$$
R_{\mathbf{X}} \approx \sum_{i=1}^{N} w_{k}^{i} \ln \frac{w_{k}^{i}}{w_{o}^{i}} .
$$

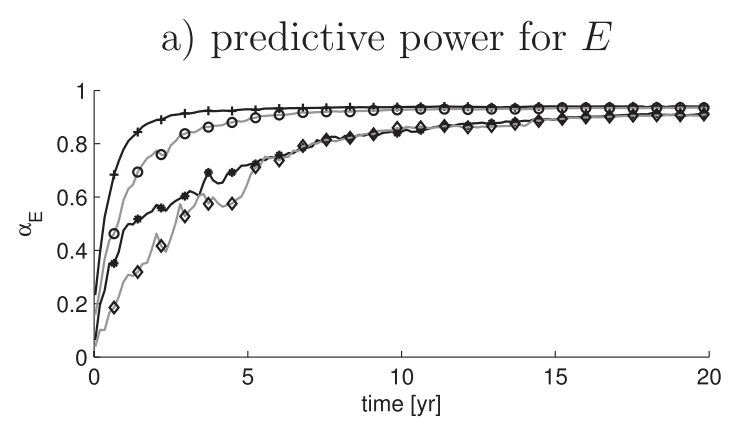

b) average potential prediction utility for $\boldsymbol{X}$

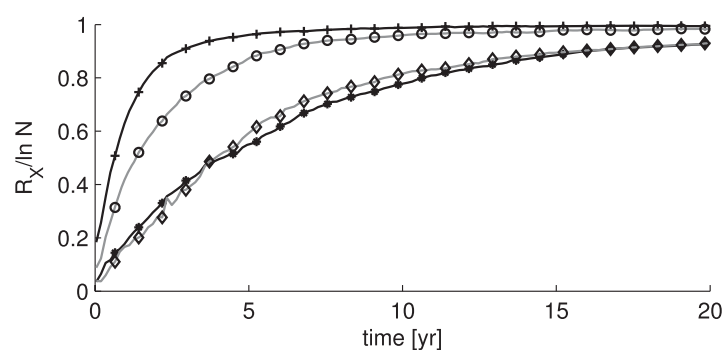

FIG. 8. (a) Average PP $\bar{\alpha}_{E}$ and (b) average PPU for the analysis calculated by repeating the algorithm with other particles being selected as the synthetic truth. The quantity is given for the four locations: A (asterisk), B (plus), C (circle), or D (diamond). 
mooring A
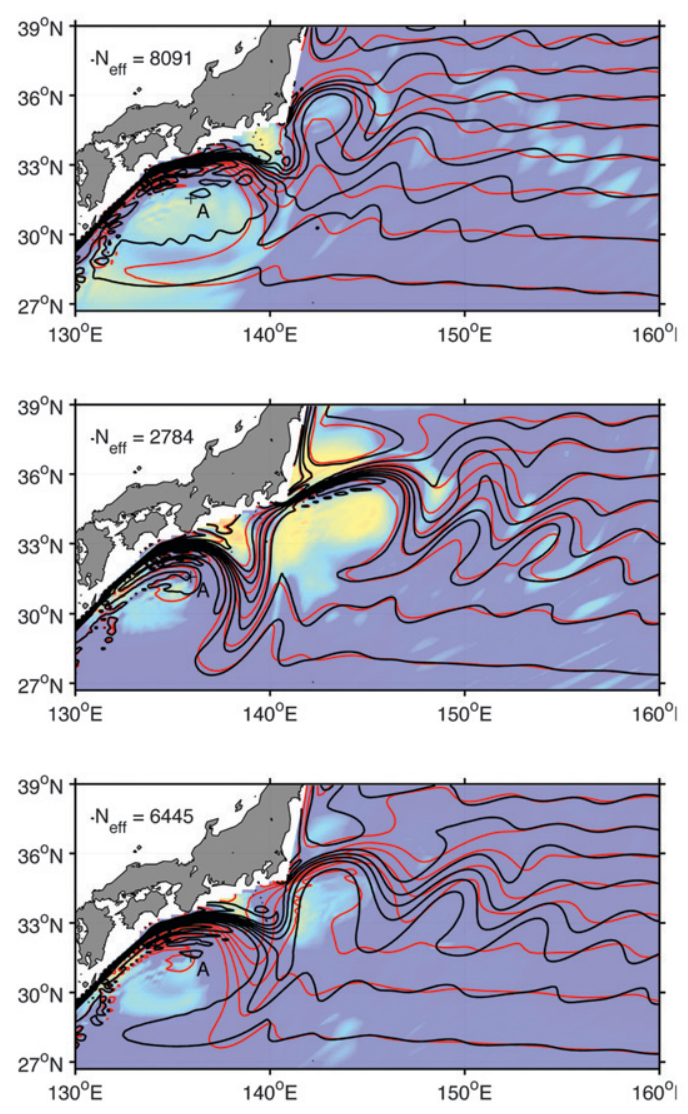

mooring B
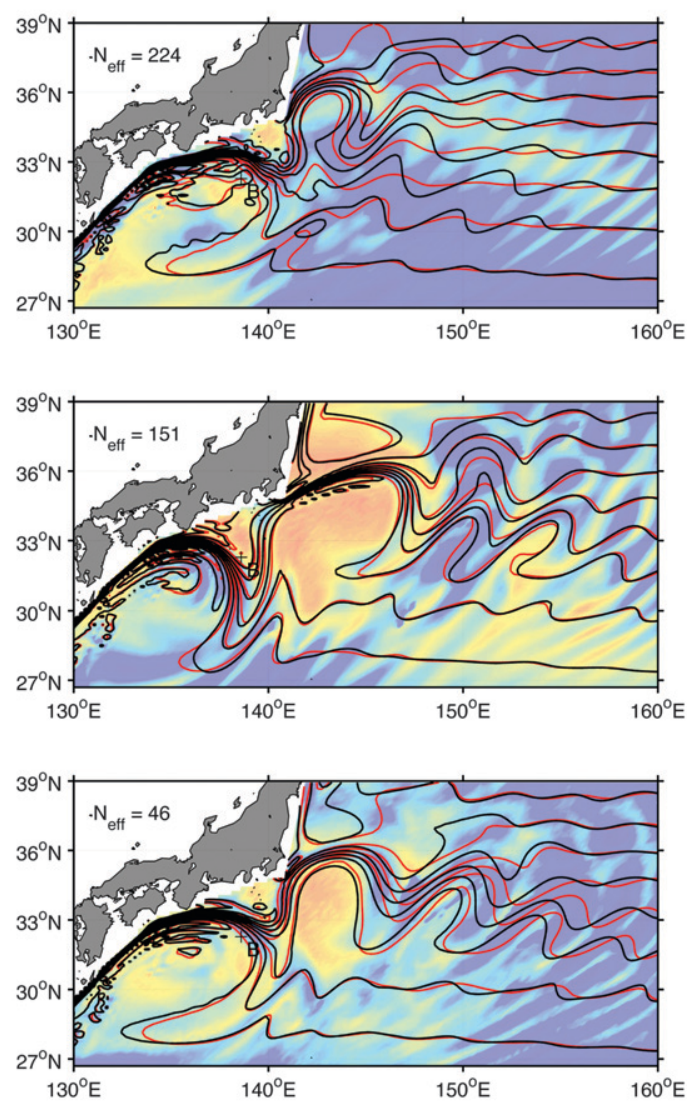

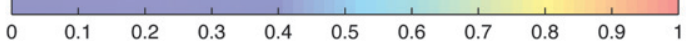

FIG. 9. One-year increase in the PP $\alpha_{Q}(\mathbf{x}, t)$. Velocity observations from mooring (left)-(right) A, B, C, and D are assimilated during one year, starting at years (top)-(bottom) 4, 8, and 12. Red contour lines depict the PV mean from the analysis. Black lines give the PV field from the synthetic truth. For each analysis, the effective number of particles is given in the top-left corner.

Note that this equation is only valid at the time an observation is made. It does not capture the decrease of $R_{\mathbf{X}}$, which occurs between observations. The practical limit for the relative entropy with an ensemble size $N$ is $R_{\mathbf{X}}=\ln N$, which we can use to normalize the PPU.

The normalized PPU averaged over 512 realizations of the synthetic truth is presented in Fig. 8b. The behavior of $\bar{R}_{E}$ is similar to $\bar{\alpha}_{E}$. The differences between the two measures mainly originate from the different sensitivity along their range. Because of the exponential term in the $\mathrm{PP}$, its sensitivity is larger in the lower part of the scale, whereas the PPU is more sensitive in the higher region of the scale. These results validate the use of $\bar{\alpha}_{E}$ to measure the impact of observations.

The degeneracy of the ensemble weights and the numerical limit to the predictive power essentially invalidate any results on predictive power after $3 \mathrm{yr}$. The sampling of the probability distribution is too poor to obtain accurate values for the entropy. Increasing the numbers of particles could alleviate both problems. A more realistic option is to restrict the analysis to a time span of one year. This essentially yields the increase of predictive power after assimilating observations over a one-year period. For this approach the ensemble time span can be reduced from 40 to $1 \mathrm{yr}$. In the following sections, we split the original ensemble $(N=512)$ into a single synthetic truth and in 20440 one-year segments. This larger ensemble can be used for an uncertainty analysis over a one-year period. Choosing this approach does not allow to perform data assimilation over a number of decades, which would give an analysis of several Kuroshio Extension path transitions. The one-year analysis can be used, however, to target the specific states of the KE 
mooring $\mathrm{C}$
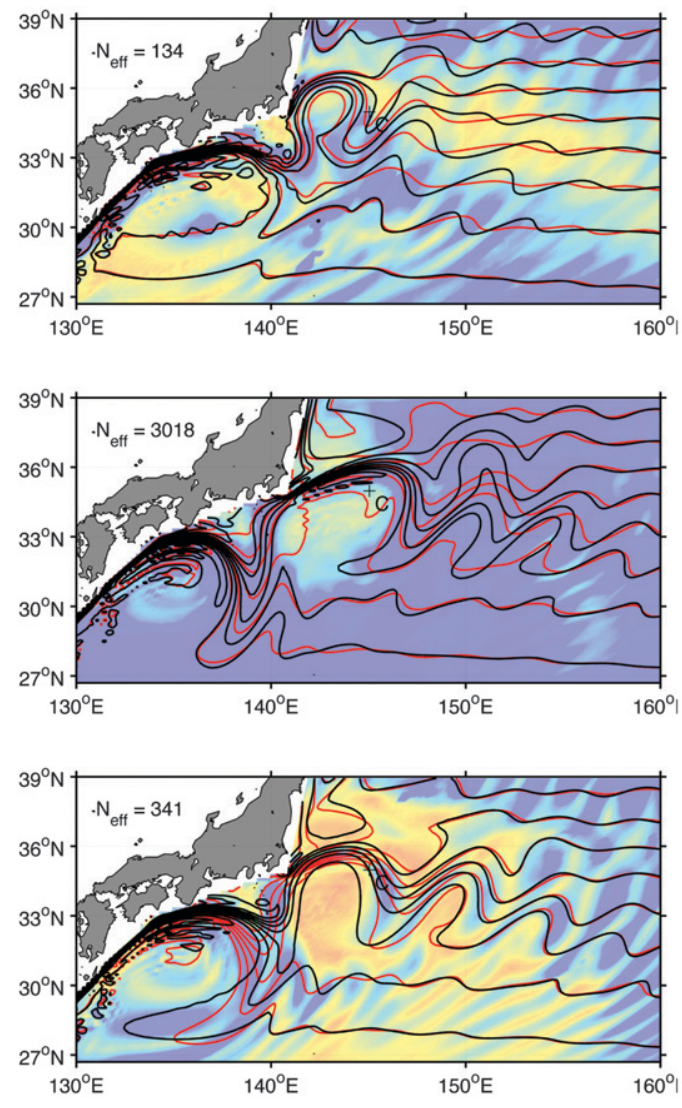

mooring D
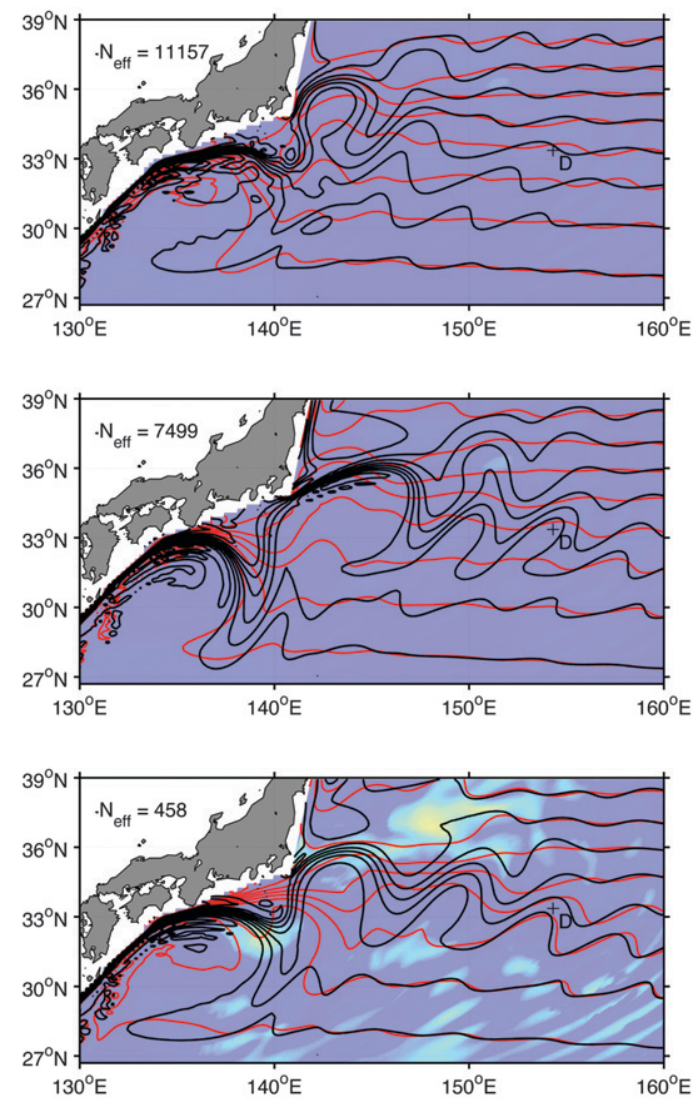

and determine which observation locations decrease the uncertainty in the analysis.

\section{Optimal monitoring of the Kuroshio Extension}

a. The spatial decrease of uncertainty in the potential vorticity

The energy of the jet $E$ is a spatially integrated signal, and hence the analysis does not give any information where the uncertainty is decreased by the assimilation of the observations. Hence, we take another approach by investigating how the uncertainty in the potential vorticity (PV) field $Q(\mathbf{x}, t)=(\zeta+f) / h$ is decreased by assimilating observations. Here, $\zeta$ is the vertical component of the relative vorticity, $\boldsymbol{\nabla} \times \mathbf{u}$. Potential vorticity is a conservative advective tracer only influenced by forcing and dissipation. Distributions of PV contain nearly all the information about the flow dynamics.
To investigate the uncertainty in the PV the predictive power is defined as

$$
\alpha_{Q}(\mathbf{x}, t)=1-\exp \left[-S_{q(Q)}(\mathbf{x}, t)+S_{p(Q)}(\mathbf{x}, t)\right] .
$$

The climatological entropy of the PV at point $\mathbf{x}, S_{q(Q)}(\mathbf{x}, t)$, is calculated using the $Q^{i}(\mathbf{x}, t)$ from the unweighted ensemble members. The climatology mean and variance of the PV are given in Fig. 4. Observations within a time span of one year are assimilated, yielding a reduction of the entropy $S_{p(Q)}(\mathbf{x}, t)$ in the weighted ensemble. This result is dependent on the time period for which observations are taken. Hence, three different time ranges, starting after 4, 8 and $12 \mathrm{yr}$, are considered (for reference, see Fig. 6). The results of the analysis are presented in Fig. 9.

The previous section revealed that, on average, velocity observations taken at locations B and C are successful for reconstructing the kinetic energy of the Kuroshio 
Extension. The study of $\alpha_{Q}(\mathbf{x}, t)$ reveals that there are differences in the performance of the moorings, depending on the state of the KE. For mooring B, we obtain good results for years 8 and 12 . In year 8 , the KE is in the high-energy state, whereas during year 12 a transition from high- to low-energy state occurs. The analysis is particularly good for the first meander of the $\mathrm{KE}$, which is also indicated by the high PP in these regions. Note that the PP is large in quiescent regions with small PV gradients. Regions where PV is more variable, like for the eastern meandering part of the KE or where the Kuroshio boundary current detaches from the Japanese coastline, are less predictable. The eastern part of the Kuroshio Extension is actually more strongly influenced by the stochastic wind forcing. Thus, part of the variability here is not correlated with the state of the $\mathrm{KE}$. When the KE has just switched to the low-energy state (year 4) the recirculation gyre is well retrieved but the reconstruction of the KE path is less successful. Low values for $\mathrm{PP}$ are thus either due to large short-time variability of the $\mathrm{KE}$ in the current state or due to dynamics that are not correlated to the observations. On the other hand, a region with high PP is correlated with the observations and the analysis has a small uncertainty.

The analysis for year 4, which uses observations from mooring $\mathrm{C}$, is more successful in retrieving the first meander of the KE. Note that the analysis for mooring B actually captures the detachment of the Kuroshio boundary current and its path quite well upstream of mooring $\mathrm{C}$. This indicates that information from mooring $\mathrm{C}$ is essential for retrieving the path of the $\mathrm{KE}$ in the meandering, low-energy phase. During the elongated-jet, high-energy state of the KE (year 8), it does not give good results because mooring $\mathrm{C}$ is located south of the KE path in a quiescent region. The success in reconstructing the eastern $\mathrm{KE}$ for the transition in year 12 is a bit surprising, because the first meander itself is not accurately recovered.

The analysis using data taken at location D yields bad results for all the stages of the KE decadal oscillation. The poor results are mainly due to the bad signal-tonoise ratio of the observed velocity signal. Data from mooring A yield bad results overall but lead to a good reconstruction of PV during the elongated-jet state of the KE (year 8). In this case, the southern recirculation gyre is stronger than in the other periods. In these other stages, the variation in the observed signal at mooring $\mathrm{A}$ is below the noise level.

\section{b. The optimal measurement location}

The four mooring locations chosen were based on the variance of the SSH field for a long climatological run. Assimilating the velocity data from these different a) the optimal spot for velocity observations

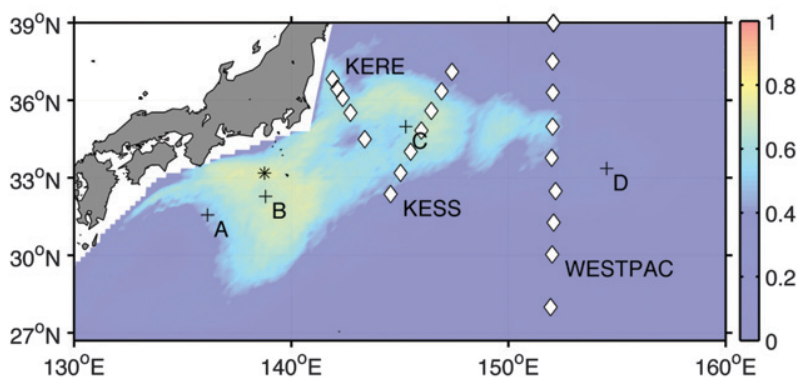

b) the optimal spot for sea-surface height observations

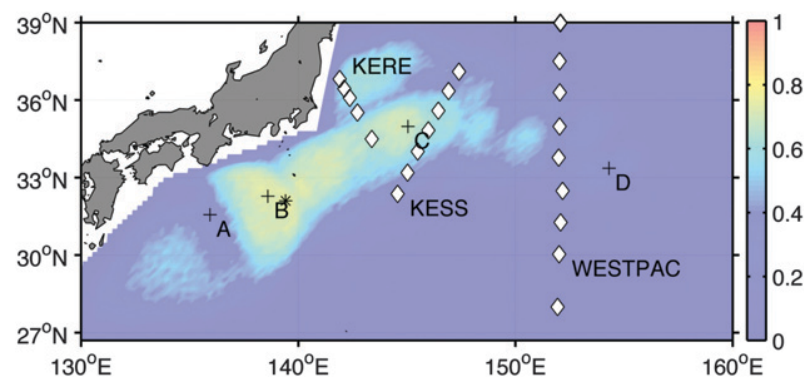

FIG. 10. A color map of the one-year increase of $\bar{\alpha}_{E}$ when (a) velocity observations or (b) SSH observations are assimilated for the given location. The star gives the optimal observation location, and the plus signs give the locations of the synthetic moorings A, B, $\mathrm{C}$, and $\mathrm{D}$. The diamonds give the position of the current meter moorings used in KERE (Hallock and Teague 1995), KESS (Donohue et al. 2008), and WESTPAC (Schmitz et al. 1982).

locations yielded varying results for reconstructing the path and kinetic energy of the KE. Mooring B yielded good results for most phases of the decadal oscillation, but its resolution of the eastern part of the KE path is somewhat lacking. Overall mooring $\mathrm{C}$ gives good reconstruction of the KE path but completely fails to capture the position of the recirculation gyre and first KE meander during the high-energy state. The opposite is true for the analysis with observations from mooring A yielding bad results overall, except for the elongatedjet state. Assimilating data over a longer period favors data from mooring B or C (Fig. 8). Most likely none of these mooring positions is the optimal location. What is the single optimal location for reconstructing the decadal oscillations in the kinetic energy of the KE? Is this location also optimal when sea surface height observations are assimilated instead of velocities?

Our answer to these questions is presented in Fig. 10. Instead of producing observations for the four locations, we apply our method to each and every grid point. Assimilating the observations for a given point yields a predictive power of $\bar{\alpha}_{E}$ (averaged over 40 synthetic truths). In Fig. 10, this value is mapped to the location where the observations originate. The optimal measurement 
location is simply the one tagged by the highest predictive power. For an analysis based on velocity observations, the optimal location is at $\left(33^{\circ} \mathrm{N}, 139^{\circ} \mathrm{E}\right)$.

Instead of using observations of the two velocity components, we can opt to assimilate sea surface height. Because we now assimilate only one variable, the observation frequency was doubled (biweekly) to obtain the same number of observations. The observational error added to the synthetic truth is $10 \mathrm{~cm} \mathrm{~s}^{-1}$. These settings result for moorings $\mathrm{A}, \mathrm{C}$, and $\mathrm{D}$ in an average increase of $\mathrm{PP}$ with a similar rate as found for velocity observations (Fig. 8). Assimilating velocity observations from location B increases the PP faster than using sea surface height. Another difference is that, by using SSH observations (instead of velocities) from mooring $\mathrm{C}$, it accurately reconstructs the PV field for year 8 , whereas it fails for year 12. When assimilating SSH observations the optimal location is at $\left(32^{\circ} \mathrm{N}, 139.5^{\circ} \mathrm{E}\right)$. For both velocity and sea surface height observations, the optimal location is located in the region where the Kuroshio boundary current detaches from the coast and has a large meander.

The question remains if the optimal observation location is optimal for both the high-energy elongated state of the KE and for the low-energy contracted state. To check this, we selected the synthetic truths that correspond to one of the KE states and calculated the average PP. The result is presented in Fig. 11 for both the low- and high-energy states. For the low-energy state, the optimal location coincides with the overall optimal location determined from Fig. 10. However, during the highenergy state, the optimal location is at $\left(36^{\circ} \mathrm{N}, 145.5^{\circ} \mathrm{E}\right)$, at the point where the energetic jet starts to meander. Measuring velocity observations in the detached Kuroshio or in the first meander of the Kuroshio Extension gives good predictive power. Note that measuring SSH along the KE path during the elongated-jet state does not give good results (Fig. 10).

Note that the optimal locations for both phases coincide with regions of strong potential vorticity gradients (see Fig. 9). These regions are susceptible to barotropic instability, and hence strong growth of perturbations can be expected. This also confirms with our finding that only a small increase of $\alpha_{Q}(\mathbf{x}, t)$ is obtained in regions with large PV gradients. For the high-energy elongated state, the largest PV gradients are south of Japan, where the Kuroshio first separates and in the first meander of the KE. Pierini (2006) argued that during this state the recirculation gyre and the $\mathrm{KE}$ are virtually isolated dynamically by the presence of the strong cyclonic meander. This view is supported by our findings that the optimal location is then in the KE itself (Fig. 11) and that observations from moorings $\mathrm{B}$ and $\mathrm{C}$ do not yield a) the optimal spot during the contracted Kuroshio Extension state

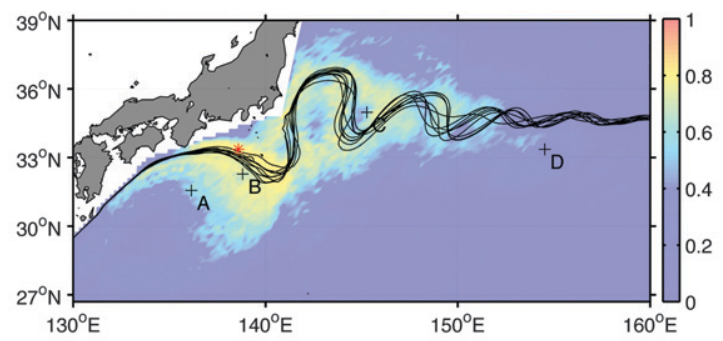

b) the optimal spot during the elongated Kuroshio Extension state

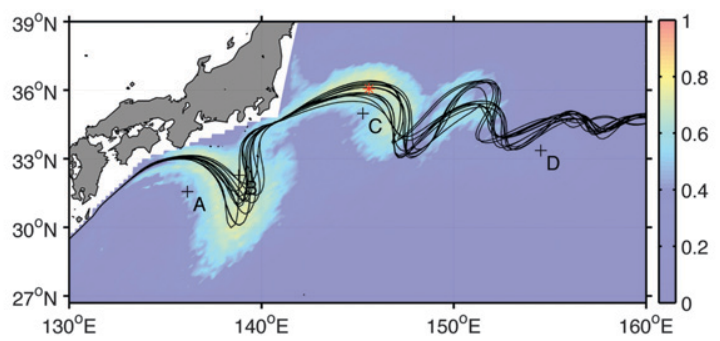

FIG. 11. A color map of the one-year increase of $\bar{\alpha}_{E}$ when velocity observations are assimilated during (a) the low-energy contracted state or (b) the high-energy jet state for the given location. For each synthetic truth, a single PV contour is used as a proxy for the KE trajectory. The star gives the optimal observation location and the plus signs give the locations of the synthetic moorings $\mathrm{A}, \mathrm{B}$, $\mathrm{C}$, and D.

a significant decrease of the uncertainty in the southern circulation gyre (Fig. 9). Note, that after the cyclonic meander the Kuroshio reattaches to the coast, where lateral friction provides a source of cyclonic PV. This is not the case in the low-energy contracted state, where the Kuroshio remains a free jet (KE) after it detaches from the coast. Hence, there is a stronger coupling between the KE and the southern circulation gyre.

\section{Summary and conclusions}

A general method is proposed to find which observation is most effective in decreasing the uncertainty in an ensemble model forecast of the Kuroshio Extension chaotic dynamics produced by a reduced-gravity shallowwater model. At all times, the unweighted ensemble represents the climatological mean and the probability distribution. As such, the ensemble itself has no predictive skill. Using a particle filter approach the weight of each ensemble member (particle) is changed according to the likelihood of the observation given the current particle state. Using the basic sequential importance sampling technique, assimilating observations is a postprocessing step. As a result, the impact of a variety of observations on the forecast uncertainty can be investigated. Assimilating a series of observations changes the 
uncertainty in the weighted ensemble. The decrease in uncertainty relative to the changeability of the climatology is measured using the predictive power. The search for an optimal observational strategy is then quantified in terms of predictive power.

On average, each subsequent observation reduces the uncertainty in the weighted ensemble. In the long term, the uncertainty converges to a value determined by the accuracy and effectiveness of the observation and by the divergence rate of the model state. The desired accuracy for sampling the long-term probability distribution prescribes the required ensemble size. In practice, the required number of model integrations is not feasible and all the weight becomes concentrated on a few particles. The traditional resolution for the degeneracy of the ensemble-resampling-would require a separate ensemble run for each set of observations. Instead, we limit the analysis of the predictive power to the short term: that is, before the ensemble degenerates.

This methodology is applied to find the optimal location to follow the path transitions of the KE in the context of a reduced-gravity model. Specifically, an identical-twin experiment is used, where the true evolution of the KE is synthetically produced by the numerical model. The kinetic energy of the KE is a good proxy to follow the chaotic decadal transitions of the KE. We have looked for the observation location that allows for the reconstruction of the kinetic energy with the lowest uncertainty, as measured by the predictive power. The optimal location for following the KE is south of the Boso Peninsula. Here, the Kuroshio typically has a large meander before entering the Pacific as a free jet. The shift of this meander and the recirculation gyre along the Japanese coastline is a clear indicator of the state of the KE. At a good measurement location, the signal-to-noise ratio has to be good and there are no elongated periods where the signal is flat. These criteria are satisfied in a region where strong PV gradients are continuously shifting.

In our study, observations of the velocity components or sea surface height at a single location are assimilated, yielding an increase of predictive power defined for a univariate quantity (the kinetic energy of the KE). The applicability of the methodology is, however, much broader. Particle filtering allows for the simultaneous assimilating multivariate observations (e.g., SSH and velocities) at multiple locations. We have seen that the success of observations in improving the forecast depends on the current state of the KE. Combining observations that are successful during different states lead to an even better observational system. Predictive power is an entropy-based measure equally suitable for measuring the uncertainty in multivariate probability distributions. This allows us to optimize the observational strategy for multiple conditions: for example, the kinetic energy of the jet and the strength of the recirculation gyre.

Optimal observation locations correspond to regions with strong potential vorticity gradients. Such PV gradients are a condition for barotropic instabilities to occur, which can lead to fast growth of perturbations. Measuring at these locations would prevent this potential growth of uncertainty. During the contracted state of the KE the strongest PV gradient is located south of Japan, where the Kuroshio detaches from the coast. The optimal location is in the elongated jet during the high-energy state of the KE. This seems to confirm that during this state the $\mathrm{KE}$ is dynamically decoupled from the southern circulation gyre (Pierini 2006).

The regional experiments of the Kuroshio Extension (WESTPAC, KERE, and KESS) were situated at different locations (Fig. 10). In the context of our reducedgravity model, the WESTPAC study at $152^{\circ} \mathrm{E}$ would be situated too far east to adequately capture the transitions of the KE. The variability related to the KE path transitions is simply too small here to yield a good signalto-noise ratio. In reality, this region is characterized by strong variability, which is caused by baroclinic instabilities. Close to Japan, where the KERE and KESS are situated, the KE is dominated by barotropic and equivalent barotropic dynamics. The KESS study captures the dynamics of the first large meander of the $\mathrm{KE}$, a region with strong PV gradients. As such, observations from KESS should capture the decadal KE transitions. For the elongated state, the path of the KE can be discerned in KERE observations. Because the path is quite stable there, it would be difficult to predict it when the transition to the contracted state occurs. Our study suggests that it is also worthwhile to monitor the detachment of the Kuroshio to determine the decadal transitions of the KE.

Acknowledgments. One of the authors (WK) is sponsored by the NSO User Support Programme under Grant ALW-GO-AO/08-14, with financial support from the Netherlands Organisation for Scientific Research (NWO). This work was sponsored by the National Computing Facilities Foundation (NCF) for the use of supercomputer facilities, with financial support from NWO. PJvL is partly sponsored by the National Centre for Earth Observation (NCEO), which is funded by the National Environment Research Council (NERC).

\section{REFERENCES}

Baker, N. L., and R. Daley, 2000: Observation and background adjoint sensitivity in the adaptive observations-targeting problem. Quart. J. Roy. Meteor. Soc., 126, 1431-1454. 
Bishop, C. H., B. J. Etherton, and S. J. Majumdar, 2001: Adaptive sampling with the ensemble transform Kalman filter. Part I: Theoretical aspects. Mon. Wea. Rev., 129, 420-436.

Cover, T., and J. Thomas, 1991: Elements of Information Theory. Wiley, $748 \mathrm{pp}$.

Deser, C., M. A. Alexander, and M. S. Timlin, 1999: Evidence for a wind-driven intensification of the Kuroshio Current Extension from the 1970 s to the 1980s. J. Climate, 12, 16971706.

Donohue, K., and Coauthors, 2008: Program studies the Kuroshio Extension. Eos, Trans. Amer. Geophys. Union, 89, 161-162.

Doucet, A., N. de Freitas, and N. Gordon, Eds., 2001: Sequential Monte Carlo Methods in Practice. Springer, 581 pp.

Griffies, S. M., and K. Bryan, 1997: Predictability of North Atlantic multidecadal climate variability. Science, 275, 181-184.

Hallock, Z. R., and W. J. Teague, 1995: Current meter observations during the Kuroshio Extension Regional Experiment. NRL Tech. Rep. MR/7332-95-7592, 120 pp.

Kagimoto, T., Y. Miyazawa, X. Guo, and H. Kawajiri, 2008: High resolution Kuroshio forecast system: Description and its applications. High Resolution Numerical Modelling of the Atmosphere and Ocean, K. Hamilton and W. Ohfuchi, Eds., Springer, 209-239.

Kleeman, R., 2002: Measuring dynamical prediction utility using relative entropy. J. Atmos. Sci., 59, 2057-2072.

— , and A. Majda, 2005: Predictability in a model of geophysical turbulence. J. Atmos. Sci., 62, 2864-2879.

Langland, R. H., 2005: Issues in targeted observations. Quart. J. Roy. Meteor. Soc., 131, 3409-3425.

Lorenz, E. N., 1975: Climatic predictability. The Physical Basis of Climate and Climate Modelling, B. Bolin, Ed., GARP Publication Series, Vol. 16, World Meteorological Organization, 130-141.

Miller, A. J., D. R. Cayan, and W. B. White, 1998: A westward intensified decadal change in the North Pacific thermocline and gyre-scale circulation. J. Climate, 11, 3112-3127.
Pierini, S., 1996: Topographic Rossby modes in the Strait of Sicily. J. Geophys. Res., 101, 6429-6440.

- 2006: A Kuroshio Extension system model study: Decadal chaotic self-sustained oscillations. J. Phys. Oceanogr., 36, 1605-1625. , 2008: On the crucial role of basin geometry in double-gyre models of the Kuroshio Extension. J. Phys. Oceanogr., 38, 1327-1333.

- H. A. Dijkstra, and A. Riccio, 2009: A nonlinear theory of the Kuroshio Extension bimodality. J. Phys. Oceanogr., 39, 2212-2229.

Qiu, B., 2002: The Kuroshio Extension system: Its large-scale variability and role in the midlatitude ocean-atmosphere interaction. J. Oceanogr., 58, 57-75.

- 2003: Kuroshio Extension variability and forcing of the Pacific decadal oscillations: Responses and potential feedback. J. Phys. Oceanogr., 33, 2465-2485.

— , and S. Chen, 2005: Variability of the Kuroshio Extension jet, recirculation gyre, and mesoscale eddies on decadal time scales. J. Phys. Oceanogr., 35, 2090-2103.

Sakov, P., and P. R. Oke, 2007: Objective array design: Application to the tropical Indian Ocean. J. Atmos. Oceanic Technol., 25, 794-807.

Schmitz, W. J., Jr., P. P. Niiler, R. L. Bernstein, and W. R. Holland, 1982: Recent long-term moored instrument observations in the western North Pacific. J. Geophys. Res., 87, 94259440 .

Schneider, T., and S. M. Griffies, 1999: A conceptual framework for predictability studies. J. Climate, 12, 3133-3155.

Shannon, C. E., 1948: A mathematical theory of communication. Bell Syst. Tech. J., 27, 370-423, 623-656.

Van Leeuwen, P. J., 2009: Particle filtering in geophysical systems. Mon. Wea. Rev., 137, 4089-4114.

$\mathrm{Xu}, \mathrm{Q} ., 2006$ : Measuring information content from observations for data assimilation: Relative entropy versus Shannon entropy difference. Tellus, 59A, 198-209. 